\section{Aderência às recomendações dietéticas do Institute of Medicine (Estados Unidos) e o seu efeito no peso durante a gestação}

\author{
Adherence to dietary recommendations by the \\ Institute of Medicine and the effect on body \\ weight during pregnancy
}

\author{
Adhesión a las recomendaciones dietéticas del \\ Institute of Medicine (Estados Unidos) y su \\ efecto en el peso durante la gestación
}

Priscila da Silva Castro ${ }^{1}$ Maria Beatriz Trindade de Castro 1 Gilberto Kac ${ }^{1}$

\footnotetext{
1 Instituto de Nutrição Josué de Castro, Universidade Federal do Rio de Janeiro. Rio de Janeiro, Brasil.

Correspondência G. Kac

Departamento de Nutrição Social e Aplicada, Instituto de Nutrição Josué de Castro, Universidade Federal do Rio de Janeiro.

Av. Carlos Chagas Filho 367, Centro de Ciências da Saúde, Bloco J, 2o andar, sala 29, Cidade Universitária, Rio de Janeiro, RJ 21941-902, Brasil. gilberto.kac@gmail.com
}

\begin{abstract}
The study's objective was to estimate the effect of adherence to dietary recommendations by the Institute of Medicine (IOM) concerning energy intake and/or macronutrient composition of diet on weight during pregnancy. This was a prospective study with observations at three time points, with a sample of 255 pregnant women. The dependent variable was gestational weight, and the independent variable was dietary adequacy according to IOM recommendations for energy and macronutrient intake, categorized as adequate versus excessive intake. Statistical analyses were performed using mixed effects models for repeated measures. Pregnant women with excessive intake were $3.59 \mathrm{~kg}$ lighter at baseline $(S E=$ $0.92 \mathrm{~kg} ; p<0.01$ ). On average, gestational weight varied by $1.83 \mathrm{~kg}(S E=0.40 \mathrm{~kg}$ ) per trimester, but pregnant women with excessive intake showed a higher weight increase of $1.70 \mathrm{~kg}(S E=0.43 \mathrm{~kg})$ during the same period as compared to women with adequate intake $(p<0.01)$. Women who adhered to IOM dietary recommendations gained less weight during pregnancy.
\end{abstract}

Food Consumption; Weight Gain; Pregnancy; Cohort Studies

\section{Resumo}

O objetivo do trabalho foi estimar o efeito que a aderência às recomendações do Institute of Medicine (IOM) dos Estados Unidos acerca da ingestão de energia elou da composição dos macronutrientes da dieta exercem no peso durante a gestação. Trata-se de estudo prospectivo com três pontos de observação que incluiu 255 gestantes. A variável dependente foi o peso aferido ao longo da gestação e a independente a adequação dietética segundo as recomendações do IOM para ingestão de energia e macronutrientes, categorizada em ingestão adequada ou excessiva. As análises estatísticas foram realizadas com modelos de efeitos mistos para medidas repetidas. As gestantes com ingestão excessiva eram $3,59 \mathrm{~kg}(E P=0,92 \mathrm{~kg})$ mais leves na linha de base $(p<0,01)$. Em média, o peso das gestantes variou $1,83 \mathrm{~kg}(E P=0,40 \mathrm{~kg})$ por trimestre, mas as gestantes com ingestão excessiva apresentaram um aumento de peso superior de 1,70kg (EP = $0,43 \mathrm{~kg}$ ), no mesmo período quando comparadas às com ingestão adequada $(p<0,01)$. Mulheres que aderiram às recomendações de ingestão dietética segundo o IOM aumentaram menos de peso durante a gestação.

Consumo de Alimentos; Ganho de Peso; Gravidez; Estudos de Coortes 


\section{Introdução}

A obesidade decorrente do ciclo reprodutivo é um importante problema de saúde pública ${ }^{1}$. A gestação é um período no qual o risco para o ganho de peso excessivo é elevado e, consequentemente, para o desenvolvimento da obesidade e de suas comorbidades, como a hipertensão arterial e a diabetes 2 .

A dieta, o nível de atividade física e algumas características sociodemográficas maternas como idade, paridade, escolaridade e raça/cor da pele estão entre os principais determinantes das alterações no peso durante a gestação 3,4 . Entre esses determinantes, a ingestão dietética e a atividade física são fatores passíveis de modificação, e devem ser considerados no aconselhamento nutricional durante o pré-natal com o objetivo de prevenir o ganho de peso gestacional excessivo. Essa prevenção é muito importante, já que está bem documentado que mulheres com ganho de peso gestacional excessivo apresentam riscos três vezes maiores de tornarem-se obesas quando comparadas àquelas com ganho de peso gestacional adequado 5 .

A alimentação equilibrada tem sido reconhecida como importante condição para uma gestação saudável. O Institute of Medicine (IOM) dos Estados Unidos recomenda que o aporte de energia não seja inferior a $1.800 \mathrm{kcal}$, enquanto a distribuição dos macronutrientes na dieta pode variar entre $10-35 \%$ de proteína, $20-35 \%$ de lipídios e $46-65 \%$ de carboidratos 6 . Castro et al. 7 analisaram o consumo de macronutrientes da dieta de 276 mulheres durante a gestação e no pós-parto, e verificaram que o percentual de macronutrientes encontrava-se dentro do recomendado pelo IOM. No entanto, as mulheres que mais diminuíram o consumo de energia do período gestacional para o pós-parto aumentaram a densidade de proteína da dieta. Em análises posteriores, confirmou-se a maior perda de peso entre as puérperas que consumiram dieta hiperproteica 8.

Em um estudo prospectivo conduzido na Alemanha com 32 gestantes, não verificou-se variação significativa na composição de macronutrientes da dieta durante a gestação ${ }^{9}$. Esse resultado evidencia que ainda não está totalmente elucidada a dinâmica das mudanças no consumo de alimentos durante a gestação, bem como o efeito da composição de macronutrientes da dieta nas alterações de peso durante a gestação. Entretanto, reconhece-se que a compreensão dessa relação pode proporcionar um aconselhamento nutricional mais efetivo durante a gravidez.

Nesse contexto, o objetivo do presente trabalho foi descrever as alterações no consumo ener- gético, dos macronutrientes (proteínas, lipídios e carboidratos), do peso e do índice de massa corporal $\left[\mathrm{IMC}=\right.$ peso $(\mathrm{kg}) /$ estatura $\left.^{2}\left(\mathrm{~m}^{2}\right)\right]$ nos três trimestres da gestação, entre mulheres com e sem sobrepeso e, sobretudo, estimar o efeito que a aderência às recomendações do IOM acerca da ingestão de energia e/ou da composição dos macronutrientes da dieta exerce no peso durante a gestação.

\section{Métodos \\ Desenho e população de estudo}

O estudo empregou um desenho do tipo coorte prospectiva. As gestantes foram arroladas durante a rotina de pré-natal no Posto Municipal de Saúde Madre Tereza de Calcutá (PS-MTC), localizado no bairro dos Bancários, Ilha do Governador, Rio de Janeiro. A captação foi realizada durante 22 meses, entre junho de 2005 e abril de 2007. Para ingressar no estudo, as mulheres convidadas atenderam aos seguintes critérios de elegibilidade: estar entre a 8a e 13a semana de gestação, ter entre 18 e 40 anos de idade, não apresentar gestação gemelar, não apresentar doenças crônicas não transmissíveis, nem doenças infecto-parasitárias e residir na área programática do estudo.

\section{Variável dependente}

A variável dependente do estudo foi o peso aferido durante a gestação em três pontos de observação: 8ạ-13ạ semanas de gestação, definido como a linha de base; 26ạ-28a semanas de gestação; e 36a-40a semanas de gestação. As gestantes foram pesadas em balança digital (Filizola Ltda., São Paulo, Brasil), sem sapatos, usando roupas leves e sem acessórios. Todas as medidas antropométricas foram realizadas por entrevistadores treinados de acordo com protocolo padronizado 10

\section{Variáveis independentes}

As informações sobre a ingestão de energia e dos macronutrientes da dieta foram obtidas em cada trimestre da gestação com base em um Questionário de Frequência de Consumo Alimentar (QFCA), previamente validado por Sichieri \& Everhart 11. As frequências de consumo referidas pelas gestantes foram transformadas em frequências diárias e, posteriormente, multiplicadas pelas quantidades de alimentos ingeridos baseadas em porções previamente estabelecidas. Para elaboração do banco de dados foram 
adotadas a Tabela Brasileira de Composição de Alimentos 12 e a tabela do Instituto Brasileiro de Geografia e Estatística 13 e, caso o alimento/nutriente não tivesse sido encontrado, utilizou-se a tabela do United States Department of Agriculture (U.S. Department of Agriculture, Agricultural Research Service. 2012. USDA National Nutrient Database for Standard Reference, release 25. http://www.ars.usda.gov/nutrientdata, acessado em 09/Jul/2012). O primeiro QFCA considerou como tempo referente o período entre a identificação positiva da gravidez até a data da primeira entrevista, qual seja, entre a 8ạ-13a semanas de gestação. Para as avaliações subsequentes considerou-se o intervalo da entrevista anterior, isto é, o tempo referente para a aplicação do segundo QFCA considerou o intervalo entre a 8ạ-13a semanas de gestação e a 26ạ-28a semanas, e o tempo referente para a aplicação do terceiro QFCA considerou o intervalo entre a 26ạ-28a semanas de gestação e a 36ạ-40a semanas.

As categorias de consumo denominadas de ingestão excessiva e ingestão adequada foram consideradas como variável independente, e foram geradas baseando-se nas recomendações do consumo de energia e da distribuição percentual dos macronutrientes da dieta aferidos na linha de base segundo as recomendações do IOM 6 .

Primeiro, calculou-se a estimativa de energia da dieta para cada gestante a partir do IMC prégestacional (IMC-PG) e dos níveis de atividade física individuais. Posteriormente, para o cálculo do consumo percentual de macronutrientes adotou-se como adequado o consumo de $1,1 \mathrm{~g}$ de proteína por $\mathrm{kg}$ de peso corporal por dia $(\mathrm{g} / \mathrm{kg} /$ dia), que somou $15 \%$ do valor energético total da dieta. Considerou-se adequado o consumo máximo de $30 \%$ de lipídios 6 .

A ingestão excessiva foi classificada quando o consumo de energia referido foi superior a $20 \%$ do recomendado pelo IOM e o consumo percentual de proteínas e lipídios fosse superior, respectivamente, a $15 \%$ e $30 \%$ do total de energia. $\mathrm{O}$ consumo percentual de macronutrientes não foi considerado independente do consumo de energia. As mulheres com consumo energético até $20 \%$ maior do que o estimado segundo o IOM, e com consumo percentual de até $15 \%$ de proteínas e 30\% de lipídios foram classificadas como ingestão adequada 6 .

\section{Covariáveis}

As covariáveis empregadas no presente estudo foram agrupadas em blocos: (i) antropométricas - estatura e IMC-PG; (ii) sociodemográficas - idade da mãe (anos), estado marital (vive em união estável, não vive em união estável), cor da pele (branca, parda ou negra), escolaridade (anos de estudos), renda familiar (quartis de renda: $\mathrm{R} \$ 0,00-500,00$; $\mathrm{R} \$ 501,00-800,00$; $\mathrm{R} \$ 801,00-$ $1.300,00$; R $1.300,00-10.000,00$ ), se trabalhou fora de casa nos últimos seis meses (sim, não), tipo de moradia (própria, outro tipo); (iii) estilo de vida - tabagismo atual (sim, não) e consumo de álcool usual (sim, não) e; (iv) reprodutivas - paridade (número de filhos). Essas variáveis foram coletadas apenas no primeiro ponto de observação quando as gestantes estavam entre 8 e 13 semanas de gestação, e foram obtidas por meio de um questionário estruturado.

A estatura foi mensurada em duplicata com estadiômetro portátil (Harpender Inc., Reino Unido). Admitiu-se uma variação máxima de $0,5 \mathrm{~cm}$ entre as duas medições. O IMC-PG foi calculado usando-se o peso medido na primeira entrevista ${ }^{1}$. Para a classificação do IMC-PG foram considerados os pontos de corte adotados pela Organização Mundial da Saúde (OMS) 14.

\section{Análise dos dados}

A amostra foi caracterizada na linha de base segundo a frequência de suas covariáveis antropométricas, sociodemográficas, do estilo de vida e reprodutivas.

Na sequência, empregou-se o modelo de efeitos mistos para avaliar a dinâmica de mudança da ingestão energética, dos macronutrientes, do peso e do IMC ao longo dos três trimestres de gestação e dos valores médios da ingestão de energia e macronutrientes da dieta na linha de base estratificando segundo o IMC $\left(\leq 24,9 ; \geq 25,0 \mathrm{~kg} / \mathrm{m}^{2}\right.$ ), que foi considerado uma variável binária tempo dependente. Em ambos os casos foram analisadas as diferenças entre essas variáveis na linha de base e o segundo e terceiro trimestres de gestação.

Posteriormente, para a análise do efeito do consumo dietético (ingestão excessiva e adequada) sobre o peso, empregou-se o modelo de efeitos mistos para medidas repetidas 15 . O peso, considerado como a variável desfecho, foi empregado como variável tempo dependente, enquanto a variável independente consumo dietético foi fixada na linha de base.

As análises foram realizadas com o programa Proc Mixed do SAS (SAS Inc., Cary, Estados Unidos). O processo de modelagem foi progressivo e elaborado em quatro etapas: (i) modelo $\mathrm{A}-$ modelo incondicional de médias que descreveu a variação do desfecho (peso) em cada nível, intra e entre indivíduos; (ii) modelo B - modelo não condicional de crescimento que incluiu a variável tempo, neste caso, o trimestre de gestação; (iii) modelo $\mathrm{C}$ - modelo condicional que incluiu 
a variável binária preditora do desfecho (ingestão adequada/ingestão excessiva); e (iv) modelo D - modelo condicional ajustado pelas covariáveis, que considerou o peso mensurado em cada ponto de observação como variável tempo dependente, e a adequação dietética como variável independente fixa no tempo. O tempo e a adequação dietética foram as únicas variáveis analisadas como efeito aleatório.

Para evitar distorção das associações pela presença de confundimento, utilizou-se o teste do qui-quadrado e o teste t de Student para verificar as associações das variáveis peso e macronutrientes da dieta (proteínas e lipídios) em grama com as variáveis sociodemográficas: idade (anos), renda (reais), escolaridade (anos) e cor da pele (branca, parda ou negra); variáveis reprodutivas: paridade (primípara e multípara); e antropométrica: IMC-PG. Todas as variáveis com valores de $\mathrm{p}<0,20$ foram selecionadas como fatores de confusão. $\mathrm{O}$ modelo $\mathrm{D}$ foi ajustado pelas seguintes covariáveis: energia, idade, cor da pele, escolaridade, paridade e IMC-PG. Todas as covariáveis contínuas foram consideradas fixas no tempo e centradas nos respectivos valores médios para melhorar a interpretação dos resultados, ou seja, permitir que variáveis com diferentes unidades de medida fossem comparáveis.

Este estudo faz parte de um projeto maior cuja pesquisa foi aprovada pelo Comitê de Ética da Secretaria Municipal de Saúde do Rio de Janeiro e está em conformidade com os princípios éticos da Resolução no 196/96 do Conselho Nacional de Saúde. Todas as gestantes foram informadas sobre o objetivo do trabalho e a participação voluntária foi condicionada à assinatura de um termo de consentimento, obtido de forma livre e esclarecida.

\section{Resultados}

O trabalho iniciou com 255 gestantes que atenderam aos critérios de elegibilidade e compareceram à primeira entrevista. Para os demais pontos de observação, compareceram, respectivamente, 150 (26ạ-28a semanas de gestação) e 95 gestantes (36a-40a semanas). As mulheres que apresentaram ingestão energética menor que $600 \mathrm{kcal} /$ dia e maior que $6.000 \mathrm{kcal} /$ dia foram excluídas da presente análise. Assim, foram estudadas as informações de 224, 133 e 83 gestantes em cada ponto de observação citado anteriormente.

$\mathrm{Na}$ linha de base, $11,6 \%$ das gestantes foram classificadas com ingestão adequada. O IMC-PG foi adequado para $60,4 \%$ das gestantes e $33,7 \%$ foram classificadas com sobrepeso ou obesidade. A maior parte das mulheres pesquisadas vivia em união $(78 \%)$ e era parda $(65,9 \%)$. As primíparas corresponderam a $49,8 \%$ da amostra, $11,8 \%$ das mulheres declararam ser fumantes e $12,5 \%$ consumiram álcool durante a gestação (Tabela 1).

As gestantes com ingestão adequada e ingestão excessiva apresentaram consumo energético na linha de base de $2.235 \mathrm{kcal}(\mathrm{DP}=568 \mathrm{kcal}) \mathrm{e}$ $3.756 \mathrm{kcal}(\mathrm{DP}=952 \mathrm{kcal})$, respectivamente.

Ao longo da gestação não houve diferença na ingestão média de energia, lipídios e carboidratos. Houve um decréscimo do consumo de proteínas de 43,6g ( $\mathrm{DP}=5,20 ; \mathrm{p}<0,001)$ entre a primeira e terceira ocasião. Inicialmente, o peso médio das mulheres foi de $61,2 \mathrm{~kg}$ ( $\mathrm{DP}=0,78 \mathrm{~kg}$ ). Em relação a linha de base, houve uma variação positiva de $3,4 \mathrm{~kg}$ no segundo (DP $=0,19 \mathrm{~kg}$; $\mathrm{p}<$ $0,001)$ e de $6,9 \mathrm{~kg}$ no terceiro trimestres $(\mathrm{DP}=$ $0,20 \mathrm{~kg} ; \mathrm{p}<0,001)$. O IMC médio na linha de base foi de $24,0 \mathrm{~kg} / \mathrm{m}^{2}\left(\mathrm{DP}=0,28 \mathrm{~kg} / \mathrm{m}^{2}\right)$ e houve um incremento médio de $1,34 \mathrm{~kg} / \mathrm{m}^{2}(\mathrm{DP}=0,08 \mathrm{~kg}$ ) $\left.\mathrm{m}^{2} ; \mathrm{p}<0,001\right)$ e $2,74 \mathrm{~kg} / \mathrm{m}^{2}\left(\mathrm{DP}=0,08 \mathrm{~kg} / \mathrm{m}^{2} ; \mathrm{p}<\right.$ $0,001)$, respectivamente, no segundo e terceiro trimestres gestacionais (Tabela 2).

A ingestão de energia ao longo da gestação não variou significativamente segundo o IMC. O consumo de macronutrientes na linha de base foi sempre maior para mulheres com IMC $\leq$ 24,9kg/m² (proteínas 171g vs. 143g; carboidratos 635g vs. 505g; lipídios 116g vs. 95g; $\mathrm{p}<0,01$ ). A diferença média da variação no consumo de proteínas foi significativamente maior entre mulheres com IMC $\geq 25 \mathrm{~kg} / \mathrm{m}^{2}$ ao longo da gestação ( $\mathrm{p}<$ 0,01 ) (Tabela 3).

O modelo A mostrou por meio da estimativa de correlação intraclasse $\left(\hat{y}=\sigma_{0}^{2} / \sigma_{0}^{2}+\sigma_{\varepsilon}^{2}\right)$ que $90 \%$ do total de alteração do peso decorreram de diferenças entre as mulheres [variação entre indivíduos $=142,7 /$ (variação entre indivíduos = $142,7$ + variação intraindivíduos $=15,89)]$. No modelo $\mathrm{B}$, por meio do cálculo do pseudo $\mathrm{R}_{\varepsilon}{ }^{2}$ (pseudo $\mathrm{R}_{\varepsilon}{ }^{2}=\sigma_{\varepsilon}{ }^{2}$ modelo $\mathrm{A}-\sigma_{\varepsilon}^{2}$ modelo $\mathrm{B} / \sigma_{\varepsilon}{ }^{2}$ modelo A), observou-se que a alteração do peso foi linear com a introdução da variável tempo, responsável por $76,4 \%$ da alteração do peso no nível 1 (variação intraindivíduos do modelo $A=15,89$ - variação intraindivíduos do modelo $\mathrm{B}=3,75$ /variação intraindivíduos do modelo $A=15,89)$. No modelo $C$, a partir da introdução da variável binária adequação do consumo (ingestão adequada/ingestão excessiva), observouse que as gestantes com ingestão excessiva eram $3,81 \mathrm{~kg}$ (erro-padrão $-\mathrm{EP}=0,96 \mathrm{~kg}$ ) mais leves na linha de base $(p<0,01)$ do que as com ingestão adequada. No entanto, as gestantes com ingestão excessiva ganharam $1,76 \mathrm{~kg}(\mathrm{EP}=0,45 \mathrm{~kg}) \mathrm{a}$ mais no mesmo período ( $\mathrm{p}<0,01)$. O modelo $\mathrm{D}$ mostrou que as gestantes com uma ingestão excessiva eram $3,59 \mathrm{~kg}(\mathrm{EP}=0,92 \mathrm{~kg})$ mais leves 
Tabela 1

Características selecionadas das 255 gestantes na linha de base (8-13 semanas gestacionais). Rio de Janeiro, Brasil, 2005-2007.

\begin{tabular}{|c|c|c|}
\hline Variáveis & $\mathbf{n}$ & $\%$ \\
\hline \multicolumn{3}{|c|}{ Adequação dietética segundo o IOM * } \\
\hline Ingestão adequada & 26 & 11,6 \\
\hline Ingestão excessiva & 198 & 88,4 \\
\hline \multicolumn{3}{|l|}{ Idade (anos) } \\
\hline $18-22$ & 85 & 33,3 \\
\hline $23-27$ & 83 & 32,5 \\
\hline $28-40$ & 87 & 34,2 \\
\hline \multicolumn{3}{|l|}{ Estatura $(\mathrm{cm})$} \\
\hline $143,1-156,6$ & 85 & 33,3 \\
\hline $156,7-162,0$ & 88 & 34,5 \\
\hline $162,1-175,8$ & 82 & 32,2 \\
\hline \multicolumn{3}{|c|}{ IMC pré-gestacional $\left(\mathrm{kg} / \mathrm{m}^{2}\right)$ ** } \\
\hline$<18,5$ & 15 & 5,9 \\
\hline $18,5-24,9$ & 154 & 60,4 \\
\hline $25,0-29,9$ & 55 & 21,6 \\
\hline$\geq 30,0$ & 31 & 12,1 \\
\hline \multicolumn{3}{|l|}{ Situação marital } \\
\hline Vive em união & 199 & 78,0 \\
\hline Não vive em união & 56 & 22,0 \\
\hline \multicolumn{3}{|c|}{ Paridade (número de filhos) } \\
\hline 0 (nulíparas) & 127 & 49,8 \\
\hline 1 (primíparas) & 79 & 31,0 \\
\hline 2-6 (multíparas) & 49 & 19,2 \\
\hline \multicolumn{3}{|c|}{ Escolaridade (anos de estudos) } \\
\hline $0-4$ & 56 & 22,0 \\
\hline $5-8$ & 69 & 27,0 \\
\hline $9-20$ & 130 & 51,0 \\
\hline \multicolumn{3}{|c|}{ Cor da pele (heteroclassificação) } \\
\hline Branca & 63 & 24,7 \\
\hline Parda & 168 & 65,9 \\
\hline Preta & 24 & 9,4 \\
\hline \multicolumn{3}{|c|}{ Trabalho fora de casa (últimos 6 meses) } \\
\hline Sim & 145 & 56,9 \\
\hline Não & 110 & 43,1 \\
\hline \multicolumn{3}{|c|}{ Renda familiar total (Reais) } \\
\hline $0-500$ & 67 & 26,4 \\
\hline $501-800$ & 62 & 24,0 \\
\hline $801-1.322$ & 63 & 24,8 \\
\hline $1.323-10.000$ & 63 & 24,8 \\
\hline \multicolumn{3}{|l|}{ Tipo de moradia } \\
\hline Própria & 156 & 61,2 \\
\hline Outros & 99 & 38,8 \\
\hline
\end{tabular}


Tabela 1 (continuação)

\begin{tabular}{lcc}
\hline Variáveis & $\mathbf{n}$ & $\%$ \\
\hline Tabagismo & & 11,8 \\
Sim & 30 & 88,2 \\
Não & 225 & 12,5 \\
Consumo de álcool & 32 & 87,5 \\
Sim & 223 & \\
Não & 22 & \\
\hline
\end{tabular}

IMC: índice de massa corporal; IOM: Institute of Medicine.

* Adequação dietética foi calculada considerando as recomendações de energia e macronutrientes do IOM 6;

** IMC classificado segundo a Organização Mundial da Saúde (OMS) 14.

\section{Tabela 2}

Valores médios da ingestão de energia e macronutrientes da dieta, peso e índice de massa corporal (IMC) na linha de base e variação no segundo e terceiro trimestres de gestação. Rio de Janeiro, Brasil, 2005-2007.

\begin{tabular}{lccccc}
\hline & $\begin{array}{c}\text { 1ㅇ trimestre } \\
\text { Média (DP) }\end{array}$ & $\begin{array}{c}\text { 2o trimestre } \\
\text { Diferença (DP) }\end{array}$ & Valor de $\mathbf{p}$ * & $\begin{array}{c}\text { 3o trimestre } \\
\text { Diferença (DP) }\end{array}$ & Valor de $p$ * \\
\hline Energia (kcal) & $3.424,6(70,23)$ & $-55,3(93,6)$ & 0,555 & $-80,5(113,2)$ & 0,478 \\
Proteína (g) & $161,6(4,35)$ & $-0,58(6,23)$ & 0,926 & $-43,6(5,20)$ & $<0,001$ \\
Carboidrato (g) & $590,8(21,06)$ & $-29,9(28,6)$ & 0,297 & $-25,2(34,7)$ & 0,468 \\
Lipídio (g) & $109,1(4,03)$ & $1,13(5,06)$ & 0,823 & $-10,5(6,16)$ & 0,088 \\
Peso (kg) & $61,2(0,78)$ & $3,40(0,19)$ & $<0,001$ & $6,94(0,20)$ & $<0,001$ \\
IMC (kg/m2) & $24,8(0,28)$ & $1,34(0,08)$ & $<0,001$ & $2,74(0,08)$ & $<0,001$ \\
\hline
\end{tabular}

DP: desvio-padrão.

* Significância da diferença da variação dos valores de energia, proteína, carboidrato, lipídio, peso e IMC na linha de base em relação ao segundo e terceiro trimestres de gestação.

Tabela 3

Valores médios da ingestão de energia e macronutrientes da dieta no primeiro trimestre e variação no segundo e terceiro trimestres de gestação segundo o índice de massa corporal (IMC) pré-gestacional. Rio de Janeiro, Brasil, 2005-2007.

\begin{tabular}{|c|c|c|c|c|c|c|}
\hline & \multirow{2}{*}{\multicolumn{2}{|c|}{$\begin{array}{c}\text { 1o trimestre } \\
\text { Médias }\end{array}$}} & \multirow{2}{*}{\multicolumn{2}{|c|}{$\begin{array}{l}\text { 2o trimestre } \\
\text { Diferenças }\end{array}$}} & \multirow{2}{*}{\multicolumn{2}{|c|}{$\begin{array}{l}\text { 3o trimestre } \\
\text { Diferenças }\end{array}$}} \\
\hline & & & & & & \\
\hline & $\leq 24,9 \mathrm{~kg} / \mathrm{m}^{2}$ & $\geq 25 \mathrm{~kg} / \mathrm{m}^{2}$ & $\leq 24,9 \mathrm{~kg} / \mathrm{m}^{2}$ & $\geq 25 \mathrm{~kg} / \mathrm{m}^{2}$ & $\leq 24,9 \mathrm{~kg} / \mathrm{m}^{2}$ & $\geq 25 \mathrm{~kg} / \mathrm{m}^{2}$ \\
\hline Energia (kcal) & 3.489 & 3.303 & 70,2 & -232 & 39,1 & -137 \\
\hline Proteína (g) & 171 & 143 * & 8,76 & $-15,6$ & 8,76 & $32,7^{\star \star}$ \\
\hline Carboidrato (g) & 635 & 505 * & $-7,93$ & $-24,5$ & $-41,8$ & 82,9 \\
\hline Lipídio (g) & 116 & 95 ** & 6,36 & $-7,88$ & 12,2 & $-12,4$ \\
\hline
\end{tabular}

* Diferença significativa $(p \leq 0,01)$ entre os valores médios do consumo de proteína, carboidrato e lipídio na linha de base segundo o IMC;

** Diferença significativa $(p \leq 0,01)$ entre as variações do consumo de proteína no tempo segundo o IMC. 
na linha de base $(p<0,01)$. Em média, o peso das gestantes variou $1,83 \mathrm{~kg}$ ( $\mathrm{EP}=0,40 \mathrm{~kg}$ ) por trimestre, mas aquelas com ingestão excessiva apresentaram um ganho de peso superior de $1,70 \mathrm{~kg}$ $(\mathrm{EP}=0,43 \mathrm{~kg})$ no mesmo período quando comparadas às com ingestão adequada $(\mathrm{p}<0,01)$. Os resultados do modelo $\mathrm{D}$ foram ajustados para as variáveis energia, idade, cor da pele, escolaridade, paridade e IMC-PG (Tabela 4).

\section{Discussão}

Os resultados do presente estudo mostram que as gestantes com ingestão de energia e composição percentual de macronutrientes da dieta ao longo da gestação dentro das recomendações do IOM ganharam menos peso do que as com ingestão excessiva. $O$ peso aumentou $1,83 \mathrm{~kg}$ por trimestre em média, mas as gestantes com ingestão excessiva ganharam mais $1,70 \mathrm{~kg}$ no mesmo período. Esses resultados se confirmaram mesmo após o ajuste para os possíveis fatores de confusão como total de energia da dieta, paridade, idade, IMC-
PG, cor da pele e escolaridade. Os resultados ressaltam a importância do acompanhamento e do monitoramento da alimentação e da variação do peso durante a gestação.

Observou-se um elevado consumo energético ao longo do seguimento e, por isto, aproximadamente $90 \%$ das gestantes estudadas foram classificadas com ingestão excessiva. Em contrapartida, somente $11,6 \%$ das gestantes apresentaram ingestão adequada na linha de base. As gestantes com ingestão adequada e ingestão excessiva apresentaram consumo energético na linha de base de $2.235 \mathrm{kcal}$ e $3.756 \mathrm{kcal}$, respectivamente. Os valores médios de consumo para gestantes com ingestão excessiva podem ser considerados elevados, mas foram semelhantes, por exemplo, aos observados em gestantes brancas, pardas e pretas que foram, respectivamente, de $2.816 \mathrm{kcal}, 3.072 \mathrm{kcal}$ e $3.192 \mathrm{kcal} 16$. No presente estudo não observou-se variação na ingestão energética ao longo dos trimestres, mas sim uma redução no consumo de proteínas quando comparada a linha de base e o terceiro trimestre. Esses resultados são semelhantes aos observados

Estimativa do coeficiente de regressão linear do modelo de medidas repetidas no tempo para alterações de peso na gestação entre mulheres com ingestão energética e composição percentual de macronutrientes adequada e excessiva, segundo a recomendação do Institute of Medicine (IOM) 6. Rio de Janeiro, Brasil, 2005-2007.

\begin{tabular}{|c|c|c|c|c|c|c|c|c|c|c|c|c|}
\hline \multirow[t]{2}{*}{ Parâmetro } & \multicolumn{3}{|c|}{ Modelo A } & \multicolumn{3}{|c|}{ Modelo B } & \multicolumn{3}{|c|}{ Modelo C } & \multicolumn{3}{|c|}{ Modelo D } \\
\hline & Beta & EP & $\begin{array}{l}\text { Valor } \\
\text { de } p\end{array}$ & Beta & EP & $\begin{array}{l}\text { Valor } \\
\text { de } p\end{array}$ & Beta & EP & $\begin{array}{l}\text { Valor } \\
\text { de } p\end{array}$ & Beta & EP & $\begin{array}{l}\text { Valor } \\
\text { de } p\end{array}$ \\
\hline \multicolumn{13}{|l|}{ Efeitos fixos } \\
\hline \multicolumn{13}{|l|}{ Intercepto } \\
\hline Peso & 63,8 & 0,77 & $<0,01$ & 57,7 & 0,80 & $<0,01$ & 61,4 & 1,20 & $<0,01$ & 60,9 & 0,90 & $<0,01$ \\
\hline Ingestão excessiva/adequada & - & - & - & - & - & - & $-3,81$ & 0,96 & $<0,01$ & $-3,59$ & 0,92 & $<0,01$ \\
\hline \multicolumn{13}{|l|}{ Taxa de variação } \\
\hline Peso*tempo & - & - & - & 3,47 & 0,10 & $<0,01$ & 1,79 & 0,42 & $<0,01$ & 1,83 & 0,40 & $<0,01$ \\
\hline Ingestão excessiva*tempo & - & - & - & - & - & - & 1,76 & 0,45 & $<0,01$ & 1,70 & 0,43 & $<0,01$ \\
\hline \multicolumn{13}{|l|}{ Componentes de variação } \\
\hline \multicolumn{13}{|l|}{ Nível 1} \\
\hline Intraindivíduos $\left(\sigma_{\varepsilon}^{2}\right)$ & 15,89 & 1,15 & $<0,01$ & 3,75 & 0,27 & $<0,01$ & 2,51 & 0,26 & $<0,01$ & 2,52 & 0,26 & $<0,01$ \\
\hline \multicolumn{13}{|l|}{ Nível 2} \\
\hline Entre indivíduos $\left(\sigma_{0}^{2}\right)$ & 142,7 & 13,38 & $<0,01$ & 152,2 & 13,68 & $<0,01$ & 155,8 & 14,54 & $<0,01$ & 21,59 & 2,17 & $<0,01$ \\
\hline \multicolumn{13}{|l|}{ Bondade do ajuste } \\
\hline Razão máxima de verossimilhança & $4.360,7$ & & & $3.815,7$ & & & $2.702,2$ & & & $2.273,8$ & & \\
\hline Critério informativo de Akaike & $4.364,7$ & & & $3.819,7$ & & & $2.706,2$ & & & $2.277,8$ & & \\
\hline
\end{tabular}

EP: erro-padrão.

Modelo A: modelo incondicional de médias; Modelo B: modelo incondicional de crescimento; Modelo C: modelo condicional (variável binária preditora do desfecho - ingestão adequada/ingestão excessiva); Modelo D: modelo condicional ajustado pelas covariáveis energia (kcal), paridade (número de filhos), idade (anos), índice de massa corporal (IMC) pré-gestacional ( $\left.\mathrm{kg} / \mathrm{m}^{2}\right)$, cor da pele (branca) e escolaridade (anos). 
por Rifas-Shiman et al. ${ }^{17}$, que investigaram 1.543 gestantes. Os autores observaram que as médias de ingestão de alimentos e nutrientes não se alteraram significativamente do primeiro para o segundo trimestre da gestação, e também verificaram uma redução no consumo de proteínas ao longo da gestação.

A dinâmica das mudanças no consumo durante a gestação ainda não foi completamente elucidada. Uma questão que parece ser unânime é o fato de que há pouca variação no consumo energético ao longo da gravidez, mas as mudanças tendem a ocorrer na estrutura da dieta, como por exemplo, o aumento ou a diminuição na proporção de algum macronutriente. Um estudo realizado na Polônia, com 119 gestantes selecionadas aleatoriamente em clínicas de prénatal, analisou as mudanças qualitativas na dieta entre o período anterior à gravidez e durante a gestação, e observou que a gravidez influenciou positivamente a qualidade geral da dieta das mulheres, que passaram a consumir mais verduras e laticínios. Ademais, a ingestão alimentar segundo grupos de alimentos foi mais próxima ao preconizado pela OMS em comparação aos hábitos alimentares anteriores à gravidez 18.

$\mathrm{O}$ consumo inadequado de macronutrientes na gestação não é incomum ${ }^{19}$. No presente estudo o consumo de proteínas diminuiu $43 g$ entre a linha de base e o terceiro trimestre da gestação. Essa redução, além de já ter sido observada em outros trabalhos 17 , pode significar um aumento no consumo de carboidratos, conforme referido por diversos autores $20,21,22$, o que pode ter contribuído para esse resultado.

Manter o ganho de peso durante a gestação dentro das recomendações do IOM não é uma tarefa fácil para a maioria das mulheres 23,24 . Essa constatação se revela especialmente importante entre mulheres que iniciam a gestação com sobrepeso/obesidade 25 , fato preocupante já que o número de mulheres em idade reprodutiva com excesso de peso tem aumentado nos últimos anos 26 .

No estudo de Nascimento \& Souza 25 , com 110 gestantes com sobrepeso ou obesidade prégestacional, verificou-se que a distribuição percentual média de macronutrientes foi de $16 \%$ de proteínas, 29\% de lipídios e 55\% de carboidratos, portanto, similar à observada no presente trabalho. Por outro lado, a ingestão energética média dessas mulheres foi de $1.736,1 \mathrm{kcal}$, inferior ao valor médio observado inclusive entre as gestantes com ingestão adequada. Uma das hipóteses é a subestimação do consumo alimentar entre mulheres com sobrepeso e obesidade pré-gestacional estudadas por Nascimento \& Souza 25. Na presente coorte, $33 \%$ das mulheres iniciaram a gestação com algum grau de excesso de peso. No entanto, essa subestimação não parece ter ocorrido, visto que as mulheres classificadas com sobrepeso apresentaram menor ingestão de energia e ganharam menos peso ao longo da gestação, mostrando coerência dos dados e adequação dos resultados. Essas gestantes eram também mais velhas e multíparas, o que pode ser devido à retenção de peso de uma gestação anterior 5 .

Stuebe et al. 24, em um estudo prospectivo com 1.388 mulheres nos Estados Unidos, exploraram associações entre dieta e atividade física com ganho de peso gestacional excessivo. Os autores concluíram que uma moderada ingestão energética, com pouca fritura, e a realização de atividade física durante a gravidez reduziram o risco de ganho de peso gestacional excessivo. Lagiou et al. 27 observaram incidência de $26 \%$ de ganho de peso gestacional insuficiente e $34 \%$ de ganho de peso gestacional excessivo. $\mathrm{O}$ consumo de gorduras foi, significativamente, menor entre as gestantes com ganho de peso gestacional insuficiente quando comparado com as com ganho de peso gestacional adequado ou excessivo. Os autores demonstraram que o aumento no ganho de peso materno estava associado com ingestão calórica, proteica e lipídica.

O presente trabalho apresenta algumas limitações, como a ocorrência de perdas de seguimento, um problema inerente aos estudos prospectivos, embora tenham sido empregadas diferentes estratégias para reduzir esta ocorrência, como ligações para confirmação das consultas agendadas, bilhetes anexados ao cartão do pré-natal para lembrá-las das datas de retorno e reforço a cada encontro da importância da participação no estudo. Apesar das perdas não observou-se um padrão seletivo em relação ao peso materno entre mulheres que ingressaram na coorte e as que alcançaram o terceiro trimestre gestacional, além de ter sido empregado procedimento de análise que considera o efeito destas perdas. Outra limitação consiste na potencial superestimativa do consumo pelo QFCA em função dos itens que compõem a lista de frequência. No período gestacional a distorção pode ser seletiva, principalmente para os alimentos mais saudáveis 28 .

Por outro lado, o QFCA vem sendo empregado em estudos epidemiológicos para mensurar o consumo usual de gestantes 29,30 , e tem sido descrito com boa reprodutibilidade e validade na aferição do consumo alimentar 31. Por último, cabe salientar que o período de referência do primeiro QFCA foi apenas o início da gestação, que compreendeu o tempo entre a descoberta da gravidez e a primeira entrevista no estudo. 


\section{Conclusão}

A presente investigação observou que as mulheres estudadas consumiram dietas altamente calóricas e que apenas $11,6 \%$ das gestantes apresentaram ingestão adequada segundo as recomendações do IOM. Entretanto, aquelas que aderiram às recomendações do IOM ganharam menos peso durante a gestação quando compa- radas com as com ingestão excessiva. Diante dessa evidência, sugere-se que a ingestão dietética dentro das recomendações do IOM pode levar ao ganho de peso gestacional adequado. Esse fato demonstra a importância do acompanhamento nutricional e orientação dietética durante o prénatal no monitoramento do ganho de peso recomendado, que pode levar à diminuição do risco na ocorrência de desfechos fetais indesejáveis.

\section{Resumen}

El objetivo del trabajo fue estimar el efecto que la adhesión a las recomendaciones del Institute of Medicine (IOM) de los Estados Unidos, acerca de la ingestión de energía y/o de la composición de los macronutrientes de la dieta, ejerce en el peso durante la gestación. Se trata de un estudio prospectivo con tres puntos de observación que incluyó a 255 gestantes. La variable dependiente fue el peso estimado lo largo de la gestación y la independiente la adecuación dietética, según las recomendaciones del IOM para la ingestión de energía y macronutrientes, categorizada como ingestión adecuada o excesiva. Los análisis estadísticos fueron realizados con modelos de efectos mixtos para medidas repetidas. Las gestantes con ingestión excesiva eran $3,59 \mathrm{~kg}(E P=$ $0,92 \mathrm{~kg})$ más leves en la línea de base $(p<0,01)$. En media, el peso de las gestantes varió $1,83 \mathrm{~kg}(E P=0,40 \mathrm{~kg})$ por trimestre, sin embargo, las gestantes con ingestión excesiva presentaron un aumento de peso superior, de $1,70 \mathrm{~kg}(E P=0,43 \mathrm{~kg})$, en el mismo período cuando se comparan con quienes tienen ingestión adecuada $(p<$ $0,01)$. Las mujeres que se adhirieron a las recomendaciones de ingestión dietética, según el IOM, aumentaron menos de peso durante la gestación.

Consumo de Alimentos; Aumento de Peso; Embarazo; Estudios de Cohortes

\section{Colaboradores}

P. S. Castro participou da concepção do projeto, análise e interpretação dos resultados, redação do artigo e aprovação final da versão publicada. M. B. T. Castro colaborou na análise e interpretação dos resultados, revisão crítica do conteúdo intelectual e aprovação final da versão publicada. G. Kac contribuiu na interpretação dos resultados, revisão crítica do conteúdo intelectual e aprovação final da versão publicada.

\section{Agradecimentos}

O projeto de pesquisa original foi financiado pelo CNPq por meio do edital CT Saúde/MCT/MS/CNPq, no 030/2004. Gilberto Kac é pesquisador nível 1D do CNPq.

\section{Conflito de interesses}

Os autores declaram que não há qualquer potencial conflito de interesse, incluindo interesses políticos e/ ou financeiros associados à realização do estudo que originou o presente artigo. 


\section{Referências}

1. Nucci LB, Duncan BB, Mengue SS, Branchtein L, Schmidt MI, Fleck ET. Assessment of weight gain during pregnancy in general prenatal care services in Brazil. Cad Saúde Pública 2001; 17:1367-74.

2. Chen Z, Du J, Shao L, Zheng L, Wu M, Ai M, et al. Prepregnancy body mass index, gestational weight gain, and pregnancy outcomes in China. Int J Gynecol Obstet 2010; 109:41-4.

3. Harizopoulou VC, Kritikos A, Papanikolaou Z, Saranti E, Vavilis D, Klonos E, et al. Maternal physical activity before and during early pregnancy as a risk factor for gestational diabetes mellitus. Acta Diabetol 2010; 47 Suppl 1:S83-9.

4. Olafsdottir AS, Skuladottir GV, Thorsdottir I, Hauksson A, Steingrimsdottir L. Maternal diet in early and late pregnancy in relation to weight gain. Int J Obes (London) 2006; 30:492-9.

5. Gunderson EP, Abrams B, Selvin S. The relative importance of gestational gain and maternal characteristics associated with the risk of becoming overweight after pregnancy. Int J Obes (London) 2000; 24:1660-8.

6. Institute of Medicine, National Academy of Sciences. Weight gain during pregnancy: reexamining the guidelines. Appendix B: supplementary information on nutritional intake. Washington DC: National Academy Press; 2009.

7. Castro MBT, Kac G, Sichieri R. Padrão de consumo alimentar em mulheres no pós-parto atendidas em um centro municipal de saúde do Rio de Janeiro, Brasil. Cad Saúde Pública 2006; 22:1159-70.

8. Castro MBT, Kac G, Leon AP, Sichieri R. High-protein diet promotes a moderate postpartum weight loss in a prospective cohort of Brazilian women. Nutrition 2009; 25:1120-8.

9. Talai Rad N, Ritterath C, Siegmund T, Wascher C Siebert G, Henrich W, et al. Longitudinal analysis of changes in energy intake and macronutrient composition during pregnancy and 6 weeks postpartum. Arch Gynecol Obstet 2011; 283:185-90.

10. Gordon CC, Chumlea WC, Roche AF. Stature, recumbent length, and weight. In: Lohman TG, Roche AF, Martorell R, editors. Anthropometric standardization reference manual. Champaign: Human Kinetics Books; 1988. p. 3-8.

11. Sichieri R, Everhart JE. Validity of a Brazilian food frequency questionnaire against dietary recalls and estimated energy intake. Nutr Res 1998; 18:1649-59.

12. Núcleo de Estudos e Pesquisas em Alimentação, Universidade Estadual de Campinas. Tabela brasileira de composição de alimentos. Campinas: Núcleo de Estudos e Pesquisas em Alimentação, Universidade Estadual de Campinas; 2011.

13. Instituto Brasileiro de Geografia e Estatística. Estudo Nacional da Despesa Familiar - ENDEF: tabela de composição de alimentos. Rio de Janeiro: Instituto Brasileiro de Geografia e Estatística; 1985.

14. World Health Organization. Physical status: the use and interpretation of anthropometry. Geneva: World Health Organization; 1995. (WHO Technical Report Series, 854).
15. Singer JD, Wilett JB. Applied longitudinal analysis: modeling change and event occurrence. New York: Oxford University Press; 2003.

16. Lacerda EMA, Kac G, Cunha CB, Leal MC. Consumo alimentar na gestação e no pós-parto segundo cor da pele no Município do Rio de Janeiro. Rev Saúde Pública 2007; 41:985-94.

17. Rifas-Shiman SL, Rich-Edwards JW, Willett WC Kleinman KP, Oken E, Gillman MW. Changes in dietary intake from the first to the second trimes ter of pregnancy. Paediatr Perinat Epidemiol 2006; 20:35-42.

18. Bojar I, Wdowiak L, Humeniuk E, Błaziak P. Change in the quality of diet during pregnancy in comparison with who and EU recommendations - environmental and sociodemographic conditions. Ann Agric Environ Med 2006; 13:281-6.

19. Sukchan P, Liabsuetrakul T, Chongsuvivatwong V, Songwathana P, Sornsrivichai V, Kuning M. Inadequacy of nutrients intake among pregnant women in the deep south of Thailand. BMC Public Health 2010; 10:572.

20. Brion MJ, Ness AR, Rogers I, Emmett P, Crib V, Smith DG, et al. Maternal macronutrient and energy intakes in pregnancy and offspring intake at $10 \mathrm{y}$ : exploring parental comparisons and prenatal effects. Am J Clin Nutr 2010; 91:748-56.

21. Halldorsson TI, Strøm M, Petersen SB, Olsen SF. Intake of artificially sweetened soft drinks and risk of preterm delivery: a prospective cohort study in 59,334 Danish pregnant women. Am J Clin Nutr 2010; 92:626-33.

22. Brekke HK, van Odijk J, Ludvigsson J. Predictors and dietary consequences of frequent intake of high-sugar, low-nutrient foods in 1-year-old children participating in the ABIS study. Br J Nutr 2007; 97:176-81.

23. Savitz DA, Stein CR, Siega-Riz AM, Herring AH. Gestational weight gain and birth outcome in relation to prepregnancy body mass index and ethnicity. Ann Epidemiol 2011; 21:78-85.

24. Stuebe AM, Oken E, Gillman MW. Associations of diet and physical activity during pregnancy with risk for excessive gestational weight gain. Am J Obstet Gynecol 2009; 201:58.

25. Nascimento E, Souza SB. Avaliação da dieta de ges tantes com sobrepeso. Rev Nutr 2002; 15:173-9.

26. Drhemer M, Duncan BB, Kac G, Schmidt MI. Association of second and third trimester weight gain in pregnancy with maternal and fetal outcomes. PloS One 8:e54704.

27. Lagiou P, Tamimi RM, Mucci LA, Adami HO, Hsieh CC, Trichopoulos D. Diet during pregnancy in relation to maternal weight gain and birth size. Eur J Clin Nutr 2004; 58:231-7.

28. Matarazzo HCZ, Marchioni DML, Figueiredo RAO, Slater Villar B, Eluf Neto J, Wünsch Filho V. Reprodutibilidade e validade do questionário de frequência de consumo alimentar utilizado em estudo caso-controle de câncer oral. Rev Bras Epidemiol 2006; 9:316-24. 
29. Marchioni DML, Latorre MR, Eluf Neto J, Wünsch Filho V, Fisberg RM. Identification of dietary patterns using factor analysis in an epidemiological study in São Paulo. São Paulo Med J 2005; 123:124-7.

30. Parra-Cabrera S, Stein AD, Wang M, Martorell R, Rivera J, Ramakrishnan U. Dietary intakes of polyunsaturated fatty acids among pregnant Mexican women. Matern Child Nutr 2011; 7:140-7.
31. Erkkola M, Karppinen M, Javanainen J, Rasanen L, Knip M, Virtanen SM. Validity and reproducibility of a food frequency questionnaire for pregnant Finnish women. Am J Epidemiol 2001;154:466-76.

Recebido em 06/Ago/2012

Versão final reapresentada em 14/Fev/2013

Aprovado em 20/Fev/2013 